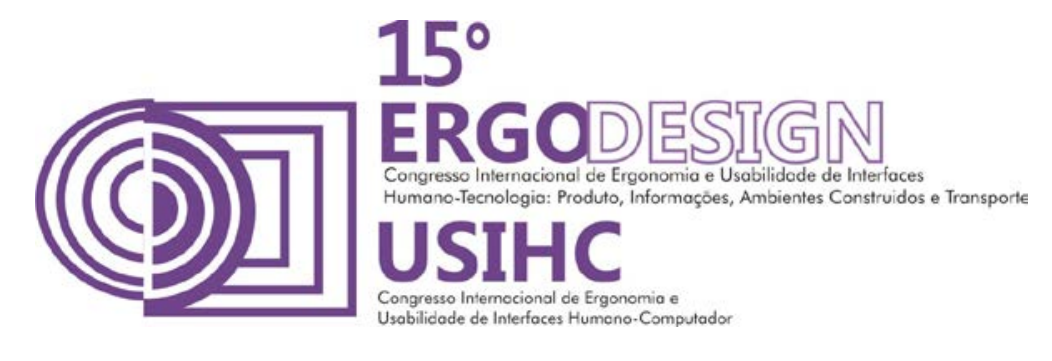

\title{
ERGONOMIA HOSPITALAR E SEU PÚBLICO-ALVO
}

\author{
MAIA, Fernanda (1); \\ FREITAS, Sydney (2) \\ (1) IFF/Fiocruz, Doutoranda em Design \\ e-mail:fernandamaiato@hotmail.br \\ (2) ESDI/UERJ, Doutor em Engenharia de produção \\ e-mail:sydneyfreitas@terra.com.br
}

\begin{abstract}
RESUMO
O objetivo deste artigo é apresentar o resultado de análise de publicações brasileiras, nos últimos 10 anos, sobre ergonomia hospitalar, que tem como foco o paciente. Levantaram-se publicações nos bancos de dados: portal de periódicos da Capes, Biblioteca Virtual em Saúde, Revista da Associação Brasileira de Ergonomia. Como resultado totalizaram-se 32 trabalhos. Destes, 30 tinham como foco de estudo 0 trabalhador de saúde, 1 tinha como foco o trabalhador de saúde e o paciente, e 1 tinha como foco os usuários (pacientes e acompanhantes). Este dado nos aponta a falta de produção científica em ergonomia hospitalar que considere o paciente.
\end{abstract}

Palavras-chave: Ergonomia, hospital, condições crônicas de saúde

\begin{abstract}
The purpose of this article is to present the result of analysis of Brazilian publications over the past 10 years, on hospital ergonomics, which focuses on the patient. Rose publications in databases: the Capes journal portal, Virtual Health Library, Revista da Associação Brasileira de Ergonomics.As a result amounted to 32 jobs. Of these, 30 were the focus of the study, 1 health worker had focused on the health worker and the patient, and 1 had focused on users (patients and accompanying persons). This points us the lack of scientific production in ergonomics consider hospital patient.
\end{abstract}

Keywords: ergonomic, hospital, chronic health conditions

\section{INTRODUÇÃO}

Atualmente, as condições crônicas de saúde como, por exemplo, a encefalopatia crônica não progressiva da infância, a broncodisplasia e a diabetes, constituem um problema de grande magnitude em âmbito mundial, sendo consideradas um grande problema de saúde pública. As condições crônicas de saúde tem como características principais a longa duração e o progresso geralmente lento. 


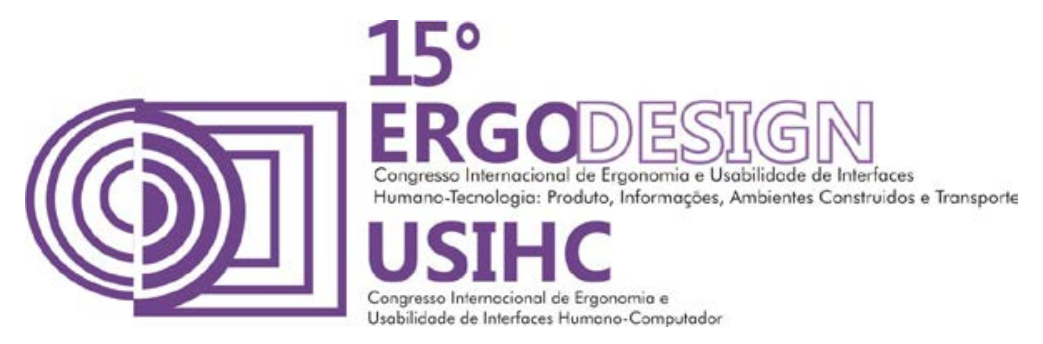

Estima-se que as condições crônicas de saúde já sejam responsáveis por 58,5\% das mortes e $45,9 \%$ da carga de doença no mundo. Seguindo a tendência mundial, no Brasil as condições crônicas de saúde são a causa de cerca de $72 \%$ das mortes e $75 \%$ dos gastos com atenção à saúde no Sistema Único de Saúde. Com o contínuo crescimento estima-se que, no ano 2020, 80\% da carga de doença dos países em desenvolvimento deverão advir de problemas crônicos. As condições crônicas de saúde constituem o desafio do setor de saúde neste século. (BRASIL, 2003; ORGANIZAÇÃO MUNDIAL DE SAÚDE, 2003)

Essa realidade se apresenta como um desafio, pois as condições crônicas de saúde apresentam um forte impacto na qualidade de vida dos indivíduos afetados, uma maior possibilidade de morte prematura e efeitos econômicos consideráveis para a família, comunidade e sociedade. (ORGANIZAÇÃO MUNDIAL DE SAÚDE, 2003)

As condições crônicas de saúde podem levar a grandes períodos de internação; a limitações funcionais como consequencia de alterações orgânicas decorrentes da patologia ou comorbidades $^{1}$ associadas; e a dependência de tecnologias ${ }^{2}$. Muitas pessoas com condições crônicas de saúde necessitam de recursos e tecnologias específicos para atenderem suas necessidades. Se considerarmos que muitas dessas pessoas passam grandes períodos internadas, seja em hospitais, em instituições ou no âmbito domiciliar (home care), devemos pensar que o seu cotidiano e suas funções e atividades se dão dentro desse contexto hospitalar.

As atividades cotidianas e a interação dessas pessoas com seu meio acontece através de sistemas e produtos com implicações ergonômicas nas suas concepções e em seus usos. Pheasant (apud MORAES, s.d., p. 02) afirma que "a ergonomia é a aplicação das informações científicas acerca do ser humano no design de objetos, sistemas e ambientes para o uso humano".

Ao entendermos a ergonomia como o estudo da interação do ser humano com seu ambiente e pensarmos no ambiente hospitalar como um local onde o usuário realiza essa interação e onde o próprio encontra-se cada vez por mais tempo (como consequência do adoecimento crônico), as atividades exercidas pelos pacientes no contexto hospitalar tornam-se alvo importante para análises ergonômicas.

\section{O HOSPITAL CONTEMPORÂNEO}

Existem relatos de instituições voltadas ao amparo dos enfermos desde a idade média, porém o hospital como instrumento terapêutico surgiu no fim do século XVIII. Apesar de muitas modificações da sociedade e da ciência desde esse período, a instituição hospitalar continua sendo um local icônico, onde a curiosidade e a repulsa, a gratidão e o medo, andam de mãos dadas.

\footnotetext{
1 "Presença ou associação de duas ou mais doenças no mesmo paciente" (Dicionário Priberam da Língua Portuguesa, 2013).

${ }^{2}$ Dependência de artefatos tecnológicos e/ou farmacológicos indispensáveis à sobrevivência.
} 


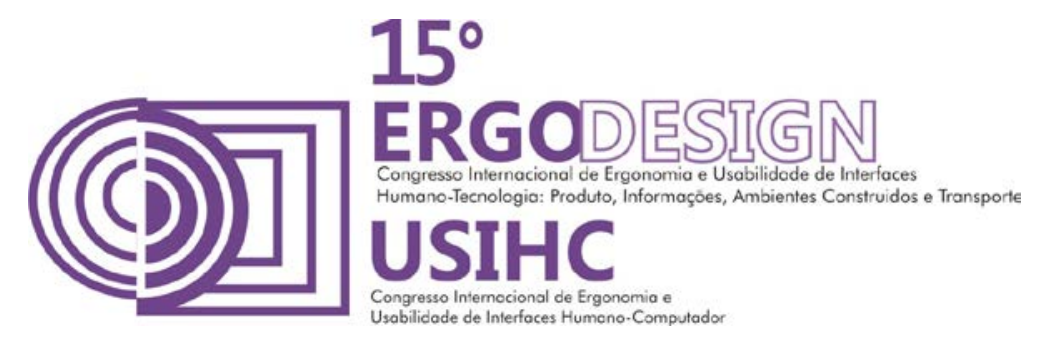

Em seu livro "Microfísica do poder", Foucault (1984) discute o nascimento do hospital moderno. Segundo o autor, até o século XVIII os hospitais eram locais onde se depositavam as pessoas doentes, principalmente os pobres, e eram geridos pelo clero. A partir de uma necessidade de ordenação do espaço hospitalar, houve uma reorganização e o início de mudanças que levaram ao hospital terapêutico. Nesse período o médico passou a ser a figura principal no hospital. A medicina via a doença como um processo decorrente da natureza, do meio em que o paciente vivia. A partir dessa premissa desenhou-se o novo modelo hospitalar onde se buscava através de hábitos, alimentação e espaços controlados uma ação terapêutica.

O ambiente hospitalar moderno, em sua essência, foi criado e pensado para tratar o doente, em sua maioria acometido por doenças agudas, e muitas vezes epidêmicas. Em um momento histórico em que o progresso levava ao aumento da população aglomerada nas grandes cidades. O hospital era o local para que o enfermo permanecesse em seu período de recuperação ou em seus últimos dias de vida, escondido dos olhos da sociedade. (ANTUNES, 1989)

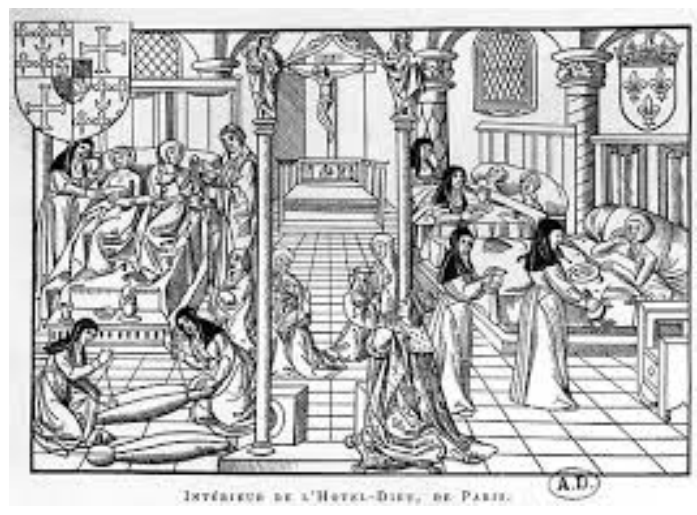

Hotel dieu, Paris, por volta de 1500

Fonte: www.art-prints-on-demands.com

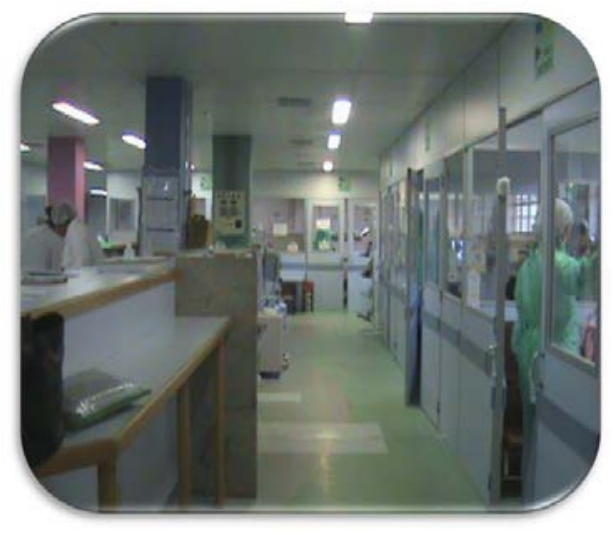

Hospital contemporâneo Foto dos autores

O hospital contemporâneo encontra-se em um momento dicotômico onde as ações são mais rápidas, porém muitas são as condições clínicas de longa duração. Desde a segunda metade do século XX vem ocorrendo uma mudança epidemiológica em nível mundial, com um crescente aumento das condições crônicas de saúde. (ORGANIZAÇÃO MUNDIAL DE SAÚDE, 2003)

"As condições crônicas constituem problemas de saúde que requerem gerenciamento contínuo por um período de vários anos ou décadas." (ORGANIZAÇÃO MUNDIAL DE SAÚDE, 2003, p. 15). As condições crônicas de saúde abarcam: condições não transmissíveis; condições transmissíveis persistentes; distúrbios mentais de longo prazo; deficiências físicas/ estruturais contínuas. Como características comuns estão a persistência e a necessidade de um nível de cuidado permanente. A Organização Mundial de Saúde (2003) destaca que as condições crônicas de saúde possuem algumas características preocupantes: 


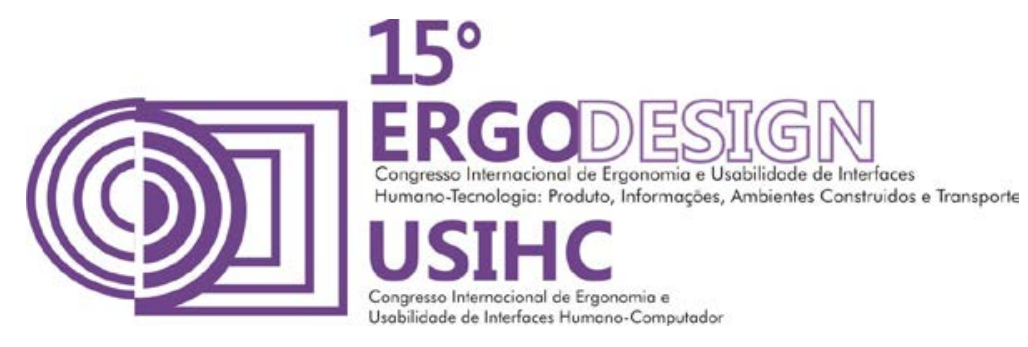

- Estão aumentando no mundo e nenhum país está imune ao impacto causado por elas.

- Representam um sério desafio para os atuais sistemas de saúde no tocante à eficiência e efetividade e desafiam as capacidades de cada país em organizar sistemas que supram as demandas iminentes.

- Causam sérias consequências econômicas e sociais em todas as regiões e ameaçam os recursos da saúde em cada país.

- Poderão ser minimizadas somente quando os líderes do governo e da saúde adotarem mudanças e inovações.

Esse quadro epidemiológico gera um cenário onde muitos pacientes ficam por períodos de dias à anos internados, ou são internados repetidas vezes. Muitos desses indivíduos tem suas funções limitadas a partir da debilidade causada pela doença ou pela necessidade de uso de aparelhos específicos que impedem suas atividades. Muitos possuem algum tipo de limitação sensorial, motora, cognitiva ou comunicacional, que leva à limitação de suas atividades cotidianas. Tais limitações, além de relacionadas as condições de saúde, estão diretamente relacionadas às dificuldades na usabilidade de instrumentos específicos da atividade pretendida (exemplo: colher, telefone, computador, etc.). Se considerarmos que muitas dessas pessoas passam grandes períodos internadas, seja em hospitais, em instituições ou no âmbito domiciliar (home care), devemos pensar que o seu cotidiano e suas funções e atividades se dão nesse contexto.

É importante ressaltar que as condições crônicas de saúde afetam indivíduos de todas as idades. Algumas condições se desenvolvem ao longo da vida, porém muitos indivíduos já nascem com condições de saúde que virão à se configurar como crônicas. Sendo assim, temos tanto adultos como crianças e adolescentes com condições crônicas de saúde inseridos no ambiente hospitalar.

Os objetos, e o meio em que encontram-se inseridos, influenciam direta e indiretamente na forma como as mais diversas atividades são realizadas no dia a dia do ser humano. Isso parece ser ainda mais evidente quando pensamos nos produtos voltados para pessoas com limitações funcionais em contextos de hospitalização. Tais indivíduos tem suas opções limitadas neste ambiente e a disponibilidade de produtos adequados as suas necessidades pode ser um fator crítico na realização de suas atividades cotidianas

\section{ERGONOMIA HOSPITALAR}

A função da ergonomia é conceder oportunidades a pessoas com diferentes potencialidades e limitações. Esse processo se dá através da adequação de tarefas, processos, sistemas, produtos e ambientes, considerando a influência de fatores do ambiente físico, social e comportamental em que cada indivíduo vive e conduz sua vida. (Baptista e Martins apud LUCIO et al, 2006; Karwowski apud MORAES e MONT`ALVÃO, 2012)

O cumprimento dos requisitos ergonômicos possibilita um ajuste adequado do sistema/produto através da maximização do conforto, satisfação e bem-estar, da garantia de segurança, da minimização dos constrangimentos, do custo humano e da carga cognitiva, psíquica e física do usuário. Da otimização do desempenho da tarefa, do rendimento e da produtividade. (MORAES e MONT`ALVÃO, 2012) 


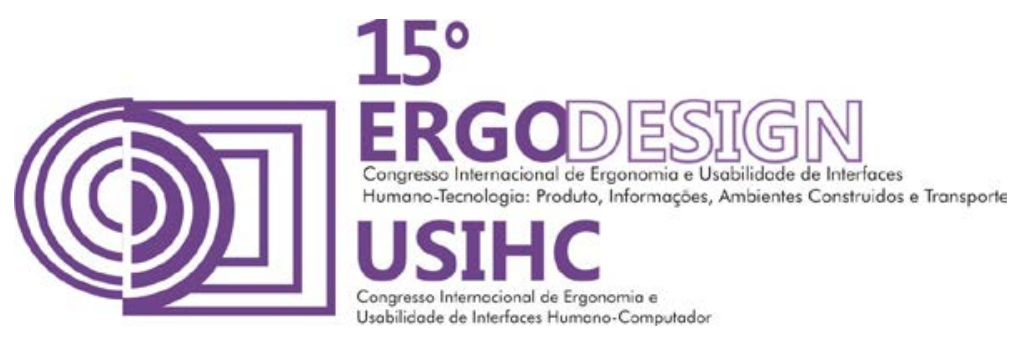

Pheasant (apud MORAES, s.d., p. 02) afirma que "a ergonomia é a aplicação das informações científicas acerca do ser humano no design de objetos, sistemas e ambientes para o uso humano" ${ }^{3}$. Sendo assim, as atividades exercidas pelos pacientes no contexto hospitalar tornamse alvo relevante para análises ergonômicas.

A Ergonomia Hospitalar tem como objetivo melhorar as condições de trabalho, conforto e segurança dos trabalhadores da área de saúde e dos pacientes. (CARDOSO e MORAES, 1998)

ELY et al (2006), ressalta essa questão ao destacar que as pesquisas ergonômicas na área de saúde vem dando maior ênfase aos problemas relacionados as atividades dos funcionários em detrimento das dos pacientes e que muitas vezes esses pacientes enfrentam dificuldades em realizar tarefas simples como consequencia da inadequação do ambiente.

Pensando que todos os produtos destinam-se a satisfazer necessidades humanas, para que esses produtos funcionem bem em suas interações com os seus usuários, devem atender a algumas características básicas: qualidade técnica, qualidade ergonômica e qualidade estética. (IIDA, 2005).

LUCIO et al (2006) destaca a necessidade de uma análise criteriosa para os equipamentos médico-hospitalares. Para o autor estes produtos devem proporcionar o máximo conforte e segurança para os seus usuários, por serem usados em situações de muito incômodo, dor e estresse.

Muito tem-se avançado na pesquisa e desenvolvimento de produtos médico-hospitalares, porém como destacam alguns autores (LUCIO, 2006; ELY et al, 2006; CARDOSO e MORAES, 1998), o foco das pesquisas ainda prioriza os profissionais de saúde e não os pacientes.

"Atualmente, apesar da maior ênfase dada aos problemas dos trabalhadores da área de saúde, alguma atenção tem sido dispensada ao paciente hospitalizado e verifica-se a aplicação da Ergonomia no projeto de utensílios e mobiliários hospitalares de uso de pacientes. São camas com acionamentos eletrônicos, cadeiras de rodas e outros produtos que amenizam os esforços do usuário ou facilitam o desenvolvimento de suas atividades. Contudo, maior atenção deve ser dispensada às necessidades do paciente, pois uma simples observação em seu cotidiano nos leva a concluir que muito há para se fazer para que o paciente possa ter melhores condições de integrar-se ao seu cotidiano. Deste modo, será possível, por exemplo, acender uma lâmpada ou usar o banheiro sem a ajuda de terceiros. A realização destas atividades simples significa para alguns pacientes, esforços enormes e muitas vezes geram constrangimentos." (CARDOSO e MORAES, 1998, p. 01)

\footnotetext{
${ }^{3}$ Texto original: Ergonomic is the application of scientific information concerning human being to the design of objects, systems and environment for human use.
} 


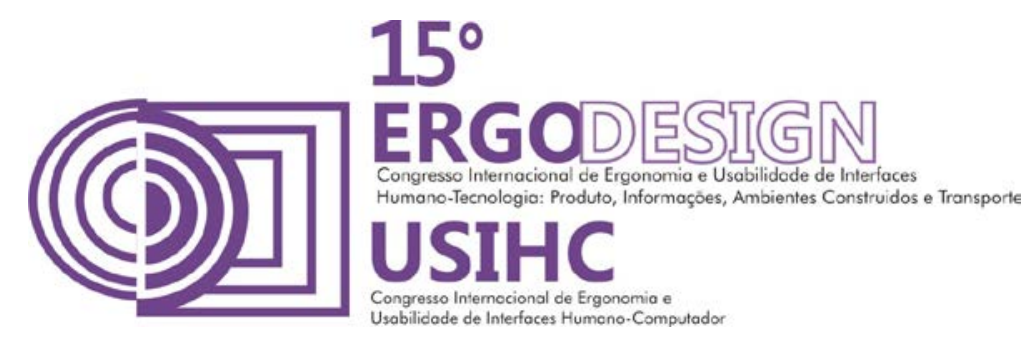

\section{QUE CAMINHO ESTAMOS TRILHANDO}

Entendemos a ergonomia hospitalar como uma área interdisciplinar e a vertente das pesquisas voltadas para o paciente, como de interesse público. Dessa forma faz-se necessário entender como vem sendo discutidas e apresentadas ao público as questões inerentes a essa área do saber.

Com o objetivo principal de entender o cenário atual sobre a produção científica na área de ergonomia hospitalar foi realizada uma revisão sistemática de publicações brasileiras, nos últimos 10 anos, no âmbito da ergonomia no ambiente hospitalar, que tenham como foco 0 paciente.

Para tal foram verificados os seguintes bancos de dados: portal de periódicos da Coordenação de Aperfeiçoamento de Pessoal de Nível Superior (Capes); Biblioteca Virtual em Saúde; Ação ergonômica - Revista da Associação Brasileira de Ergonomia. O primeiro banco de dados foi escolhido por sua relevância e abrangência na área de pesquisa, possibilitando que tivéssemos acesso a estudos de diferentes áreas científicas. O segundo banco de dados foi escolhido por sua representatividade da área de saúde, área essa fortemente associada ao tema em questão. O terceiro foi escolhido por sua relevância na área de ergonomia no Brasil.

Foram pesquisados os termos "ergonomia" e "hospital" conjuntamente nas categorias título, resumo e assunto. Os limites foram: ser em português e terem sido publicados nos últimos 10 anos. Foram analisados os resultados e excluídos os que apareciam por vezes repetidas ou que não estavam dentro do escopo do tema "ergonomia hospitalar".

Como resultado totalizaram-se 32 trabalhos. Dentre eles, 23 eram artigos científicos e 9 eram dissertações. Deste total, 30 tinham como foco de estudo o trabalhador de saúde, 1 tinha como foco o trabalhador de saúde e o paciente, e 1 tinha como foco os usuários (pacientes e acompanhantes). Este dado nos aponta uma gritante falta de produção científica em ergonomia hospitalar que considere o paciente.

Destacou-se também o fato, que dentre os poucos que incluíram o paciente como foco da análise ergonômica (2 apenas), nenhum teve como alvo a clientela infantil.

Em análise quanto às bases de publicação, verificou-se que as dissertações foram apresentadas na Faculdade de Enfermagem da Universidade do Estado do Rio de Janeiro (6 trabalhos), Na Escola Nacional de Saúde Pública Sergio Arôuca - ENSP (2 trabalhos), no Instituto Alberto Luiz Coimbra de Pós-Graduação e Pesquisa de Engenharia - COPPE (1 trabalho).

Quanto às revistas científicas, a grande maioria foi na área de saúde (17 trabalhos), sendo as revistas de publicação: Revista de Nutrição (2 trabalhos), Revista de Pesquisa Cuidado é Fundamental (2 trabalhos), Revista Ciência e Saúde coletiva (3 trabalhos), Journal of health informatics (1 trabalho), Revista Brasileira de Saúde Ocupacional (1 trabalho), Revista de Enfermagem da Universidade Federal de Pernambuco (1 trabalho), Revista Mineira de Enfermagem (1 trabalho), Revista Acta scientiarum health sciences (1 trabalho), Revista Paraense de Medicina (1 trabalho), Revista Brasileira de Enfermagem (2 trabalhos), Revista Mundo Saúde (1 trabalho), Revista de Enfermagem da Universidade do Estado do Rio de Janeiro (1 trabalho). Os artigos restantes foram publicados na Revista Gestão e Produção (1 trabalho), Revista Ação Ergonômica da Associação Brasileira de Ergonomia (2 trabalhos), Alfa - 


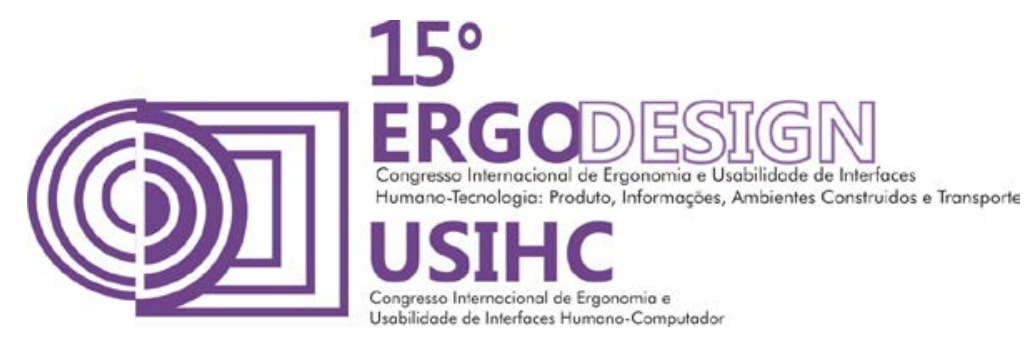

Revista de Linguistica (1 trabalho), Cadernos de Psicologia Social do Trabalho (1 trabalho), Revista Produção da Associação Brasileira de Engenharia de Produção (1 trabalho). A partir dessa análise é possível observarmos que a ergonomia hospitalar é um campo híbrido do saber, onde várias categorias científicas se encontram. Além disso, muitas são as publicações na área de enfermagem, o que em parte, podem justificar o fato da maioria das publicações ter como fonte de pesquisa as análises e requisitos ergonômicos visando a saúde, a segurança e o conforto do profissional de enfermagem.

Quanto aos artigos que tinham como foco o usuário, o primeiro intitulava-se "avaliação do desempenho do layout e da sinalização de uma unidade hospitalar" (RANGEL, M. e MONT'ALVÃO, C., 2011) e foi parte de uma pesquisa de mestrado em Design. O artigo parte da premissa de que a localização dentro de um ambiente hospitalar pode ser um ponto gerador de angustia para pacientes e usuários, e como tal deve ser analisado e desenvolvido de forma criteriosa. O estudo aponta a necessidade de atenção a questões como arranjo espacial e legibilidade no hospital pesquisado e pode servir de base para estudos similares em outras instituições.

O segundo artigo intitulado "avaliação ergonômica de cadeira de rodas utilizada no transporte de pacientes em hospital" (JORGE, S. S. e ALEXANDRE, N. M. C., 2005) realizou a avaliação de cadeiras de rodas para transporte intra-hospitalar. Os sujeitos da pesquisa foram profissionais de saúde que conduziam as cadeiras e usuários que eram conduzidos nas mesmas. Verificou-se uma inadequação do artefato, apontado principalmente pelos trabalhadores. Os autores ressaltam a importância de novas pesquisas na área para 0 fornecimento de subsídios para os processos de seleção e aquisição de materiais e objetos de uso hospitalar.

\section{CONSIDERAÇÕES}

A ergonomia trata da relação homem-tarefa-objeto-sistema. Em um ambiente hospitalar essa relação é observada nas atividades dos profissionais, usuários e acompanhantes. Em uma situação de limitação como a do paciente internado, as recomendações ergonômicas podem não só facilitar a atividade gerando maior conforto, segurança e satisfação, como ser o fator diferencial na possibilidade do usuário realizar ou não a tarefa. O desenvolvimento de produtos voltados adequados ergonomicamente passa a ser um fator determinante no nível da capacidade, independência e consequente qualidade de vida do indivíduo com limitações funcionais.

A partir de uma análise sistemática da literatura pudemos observar que há um número significativo de publicações na área de ergonomia hospitalar, mas as publicações nessa área que tenham como foco o paciente são escassas.

Muito se discute sobre inclusão, qualidade de vida e promoção de saúde no ambiente hospitalar, porém pouco se pensa nesses tópicos associados ao desenvolvimento de produtos. Faz-se necessário o incentivo de pesquisas que visem a avaliação ergonômica das atividades exercidas pelos pacientes para que possam ser pensados produtos e sistemas mais adequados para esse cenário. 


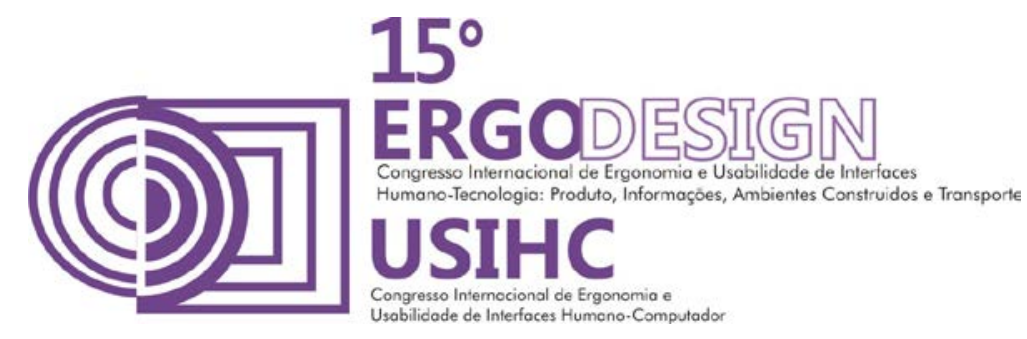

\section{REFERÊNCIAS BIBLIOGRÁFICAS}

ANTUNES, J. L. Por uma geografia hospitalar. Tempo Social; Revista de Sociologia da Universidade de São Paulo, São Paulo, 1(1): 227-234, 1.sem. 1989.

CARDOSO, V.M.B.; MORAES, A. Ergonomia hospitalar: atividades de alimentação e deficiências dos equipamentos disponíveis. XVIII Encontro Nacional de Engenharia de produção e IV Congresso Internacional de Engenharia Industrial. Rio de Janeiro. 1998.

ELY, V. M. B.; CAVALCANTI, P. B.; BEGROW, A. P.; DENK, E. C. Estudo de caso de unidade de internação hospitalar com foco na percepção e comportamento dos usuários. Anais do $14^{\circ}$ Congresso Brasileiro de Ergonomia. Curitiba. 2006

FOUCAULT, M. O nascimento da clínica. Ed. Forense Universitária. 6ª Ed. Rio de Janeiro. 2004.

IIDA, I. Ergonomia: projeto e produção. 2. ed. [S.I.]: Edgard Blücher, 2005.

JORGE, S. S.; ALEXANDRE, N. M. C. Avaliação ergonômica de cadeira de rodas utilizada no transporte de pacientes em hospital. Revista de Enfermagem da Universidade do Estado do Rio de Janeiro. 2005; 13:181-7

LUCIO, C. C.; PASCHOARELLI, L. C.; RAZZA, B. M. A importância do design universal aplicado a equipamentos médico-hospitalares: uma revisão. Anais do $14^{\circ}$ Congresso Brasileiro de Ergonomia. Curitiba. 2006

MORAES, A. M. Ergonomics \& Design, Ergonomic Design, Ergodesign.[s.d.] Disponível em: http://www.ergodesignforum.com/documents/papiers/lundi/ DeMoraesAnamaria_050509.pdf . Acesso em: 13 de Junho de 2010.

MORAES, A.; MONT`ALVÃO, C. Ergonomia: Conceitos e aplicações. Ed. 2AB. Teresópolis. 2012.

ORGANIZAÇÃO MUNDIAL DA SAÚDE. Cuidados inovadores para condições crônicas Componentes estruturais de ação: relatório mundial. Brasília, 2003.

RANGEL, M.; MONT`ALVÃO, C. Avaliação do desempenho do layout e da sinalização de uma unidade Hospitalar. Ação ergonômica. volume 6, número 1. 2011 